\section{The Questions of Tenure}

Edited by Richard P. Chait Harvard University Press, \$35.00, 352 pp, 2002

\author{
Reviewed by Sanjay K. Nigam \\ Departments of Pediatrics, Medicine and \\ Cellular Molecular Medicine \\ University of California, San Diego \\ La Jolla, California, USA
}

Ralph Waldo Emerson, whose ideas have exerted immense influence on American academics, famously argued, "Whoso would be a man, must be a nonconformist." There are only a few places left where nonconformity-or at least the possibility of it (translated into creativity and intellectual autonomy)_remains protected. Universities are supposed to such places, and tenure is a major mechanism by which this protection is accomplished.

Concepts like creativity and intellectual autonomy are nebulous, not clearly ascertainable by committees influenced by university politics or administrators with a 'research dollars per square foot' perspective. In theory, tenure serves as a shield for the innovative academician who has already proven her or himself by traditional CV (curriculum vitae) criteria. They have played the game, but now the careerist $\mathrm{CV}$ approach required to obtain tenure is perhaps their greatest impediment to creative work. With tenure, they can afford to take intellectual risks, fearlessly cross disciplinary boundaries, even if their peers don't quite understand what they are doing or why. They can even make mistakes and then try again.

So goes one professorial argument. But, as is pointed out in The Questions of Tenure, a compilation of articles edited by Richard Chait, the criteria for tenure vary greatly from institution to institution. Rates of tenure may be as low as $10 \%$ at the country's leading private universities and as high as $95 \%$ at some small colleges. Clearly the concept of what constitutes tenurable achievement means different things at different places. At certain institutions, tenure is a reward for truly extraordinary accomplishment whereas at others it is part of the natural order, mere certification that a minimum standard has been met. Moreover, tenure is not only awarded by different criteria at different places, its substantive value is also different at different places.

These two somewhat unsurprising points are among the main conclusions drawn by authors in The Questions of Tenure, a reasonably thorough discussion of the current state of tenure in American colleges and research institutions. Though not the juiciest read, it is a useful contribution that examines the concept of tenure, both theoretically and operationally, and the debate over reform. The stated intention is to provide data rather than rhetoric. The book may be of particular value to policy makers and others who are under the misconception that tenure is uniform among institutions. The core issue of "whether traditional tenure, basically developed by white males for white males, remains appropriate to contemporary conditions," is analyzed from multiple angles. The questions of faculty autonomy, probationary periods and post-tenure reviews are dealt with at some length as are the changing demographics of the socalled academy: the increase in part-timers, women and aging tenured faculty who can block the entry of new tenure-track faculty simply because such positions are limited.

The strength of the book as a whole is that it is mostly written by senior academics who have participated in the debate for some time and have well consid-

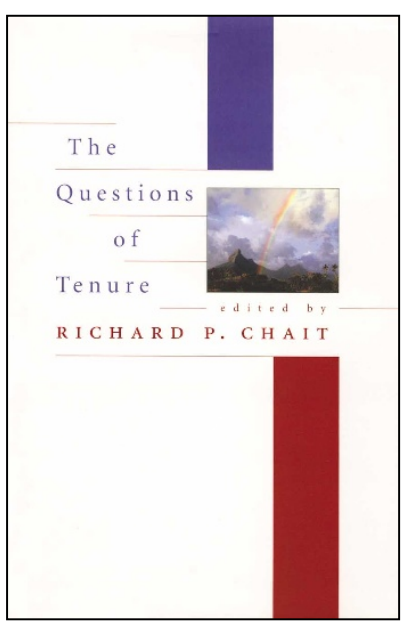

this regard, it is worth noting that some trustees and administrators who have seriously thought of reforming tenure have concluded that the costs, political and financial, do not justify the imagined gains. A chapter on the question of whether colleges can effectively recruit without tenure makes the case that, while prospective faculty strongly prefer tenure-track positions, an attractive geographical location and appealing balance of work at a strong department can sway applicants to accept non-tenure track positions. Although this is not surprising, perhaps the obvious needs to be stated.

Occasionally, an unintentionally amusing conclusion emerges. A personal favorite is: "Administrators in search of power should seek senior positions at small, comparatively impoverished colleges."

Chait's summary, a chapter called "Gleanings", is quite good. Again he emphasizes the importance of context, and the fact that, despite the data, there are no clear answers. As he states, "Detractors can properly label tenure an impediment to strategy, efficiency and creativity, and proponents can credibly argue to the contrary. These are all correct assertions when qualified by context. The only mistaken position would be to categorically condemn or defend tenure as if there were uniform practices and results."

The question of tenure has to do with ered views; this is, rather obviously, its weakness, too. Few surprises emerge. If, as contended, tenure is "the abortion issue of the academy," in some contributions one gets the sense that universities and colleges must be boring places, and there is really no issue that comes close to being so contentious. Nevertheless, there are nice explorations of what constitutes scholarship, of collegial versus managerial cultures, and what circumstances might induce a senior faculty member to relinquish tenure. The wisdom and practicability of radically revising tenure policies is also addressed. In what the academy is all about and whether, in an era when universities and industry continue to embrace, the distinctions between what it means to be a member of the academy as opposed to the corporate world ought to be preserved. But that is only part of it. Though he does not investigate the issue deeply enough, Chait points out, "On or just below the surface lie issues of values and power...Who should make the rules? and Who should make the decisions?" A more Foucault-like analysis of power relationships within the university setting might have taken this book to another level. 